\title{
Impact of peer review on reports of randomised trials published in open peer review journals: retrospective before and after study
}

\section{Sally Hopewell senior research fellow ${ }^{12}$, Gary S Collins senior research fellow ${ }^{1}$, Isabelle Boutron professor ${ }^{2}$, Ly-Mee Yu senior medical statistician ${ }^{1}$, Jonathan Cook associate professor ${ }^{13}$, Milensu Shanyinde medical statistician ${ }^{1}$, Rose Wharton medical statistician ${ }^{1}$, Larissa Shamseer PhD student ${ }^{4}$, Douglas G Altman director ${ }^{1}$}

${ }^{1}$ Centre for Statistics in Medicine, University of Oxford, UK; ${ }^{2}$ Centre d'Epidémiologie Clinique, Université Paris Descartes, INSERM U1153, France; ${ }^{3}$ Nuffield Department of Orthopaedics, Rheumatology and Musculoskeletal Sciences, University of Oxford, UK; ${ }^{4}$ Ottawa Hospital Research Institute, Canada

\begin{abstract}
Objective To investigate the effectiveness of open peer review as a mechanism to improve the reporting of randomised trials published in biomedical journals.

Design Retrospective before and after study.

Setting BioMed Central series medical journals.

Sample 93 primary reports of randomised trials published in $B M C$-series medical journals in 2012.

Main outcome measures Changes to the reporting of methodological aspects of randomised trials in manuscripts after peer review, based on the CONSORT checklist, corresponding peer reviewer reports, the type of changes requested, and the extent to which authors adhered to these requests.

Results Of the 93 trial reports, $38 \%(n=35)$ did not describe the method of random sequence generation, $54 \%(n=50)$ concealment of allocation sequence, $50 \%(n=46)$ whether the study was blinded, $34 \%(n=32)$ the sample size calculation, $35 \%(n=33)$ specification of primary and secondary outcomes, $55 \%(n=51)$ results for the primary outcome, and $90 \%(n=84)$ details of the trial protocol. The number of changes between manuscript versions was relatively small; most involved adding new information or altering existing information. Most changes requested by peer reviewers had a positive impact on the reporting of the final manuscript-for example, adding or clarifying randomisation and blinding $(n=27)$, sample size $(n=15)$, primary and secondary outcomes $(n=16)$, results for primary or secondary outcomes $(n=14)$, and toning down conclusions to reflect the results $(n=27)$. Some changes requested by
\end{abstract}

peer reviewers, however, had a negative impact, such as adding additional unplanned analyses $(n=15)$.

Conclusion Peer reviewers fail to detect important deficiencies in reporting of the methods and results of randomised trials. The number of these changes requested by peer reviewers was relatively small. have a negative impact on reporting in the final publication.

\section{Introduction}

The International Committee of Medical Journal Editors defines peer review as the "critical assessment of manuscripts submitted to journals by experts who are not part of the editorial staff."1 The practise of peer reviewing manuscripts has been around for more than 200 years and today editorial peer review is used almost universally by scientific journals as a tool to assess and improve the quality of submissions to biomedical journals. ${ }^{2}$ One of the main aims of peer review is to improve the quality and transparency of a publication by checking that the reported research has been carried out correctly and that the results presented have been interpreted appropriately. ${ }^{3}$ Clinical decisions are then made on the basis of this research evidence, so if it is misleading, by being incomplete, inaccurate, or poorly reported this has a direct impact on patient care.

Traditionally, the process of peer review has been carried out "blinded" so that the identity of the peer reviewers is concealed from the authors and the comments made by the peer reviewers are never available in the public domain. Some journals have since switched to a process of open peer review, ${ }^{4}$ whereby the Although most had a positive impact, some were inappropriate and could 
identity of the peer reviewers is disclosed and, in some instances, the peer reviewer's comments are included alongside the published article. However, despite several studies assessing the effectiveness of the peer review process, and its wide acceptance by the scientific community, little is known about its impact on the quality of reporting of the published research. ${ }^{5}$ Given the wealth of evidence on the poor and inadequate reporting in published research activities, particularly the reporting of randomised trials, ${ }^{6}$ peer reviewers may be failing to detect important deficiencies in the scientific literature.

We investigated the effectiveness of open peer review as a mechanism to improve the reporting of randomised trials published in biomedical journals. In particular, we examined the extent of changes made to the reporting of methodological aspects of manuscripts of randomised trials after peer review, the type of changes requested by peer reviewers, and the extent to which authors adhere to these requests.

\section{Methods}

\section{Sample selection}

We searched the US National Library of Medicine's PubMed database to identify all primary reports of randomised trials published in the BioMed Central series medical journals (see supplementary appendix 1 for search strategy) in 2012 and indexed with the publication type "Randomized Controlled Trial" (search as of 28 May 2013). One reviewer (SH) screened the titles and abstracts of all retrieved reports to exclude any obvious reports of non-trials. A copy of the full article was then obtained for all non-excluded reports, and two reviewers assessed each article for eligibility. We chose to use the $B M C$-series medical journals as they operate a system of open peer review whereby all submitted versions of a manuscript are published along with the corresponding peer reviewers' comments and authors' responses. ${ }^{7}$ Manuscripts submitted to $B M C$-series medical journals are typically handled by external academic editors, in house editors, or a combination of both at different stages in the manuscript submission process. Comments made by the external academic editors are not usually published alongside the submitted manuscript. Journals included in the $B M C$-series medical journals also endorse the CONSORT (Consolidated Standards of Reporting Trials) statement. ${ }^{8}$

\section{Eligibility criteria}

We included all primary reports (that is, those reporting the main study outcome) of randomised trials, defined as a prospective study assessing healthcare interventions in human participants who were randomly allocated to study groups. We included all studies of parallel group, crossover, cluster, factorial, and split body design. We excluded protocols of randomised trials, secondary analyses, systematic reviews, methodological studies, pilot studies, and early phase trials (for example, phase 1).

\section{Data extraction}

Eight reviewers working in pairs extracted data. Each pair independently extracted data from eligible reports; any differences between reviewers were resolved by discussion, with the involvement of an arbitrator if necessary. To ensure consistency between reviewers we first piloted the data extraction form. We discussed any disparities in the interpretation and modified the data extraction form accordingly. By accessing the prepublication history from each report (using the URL cited at the end of each published article) we recorded the number of submitted versions of the manuscript and the date of the original submission, final submission, and published article. For each eligible report we used the "compare documents" function in PDF Converter (Enterprise 7) to determine differences in reporting between the original submitted version of the manuscript and the final submitted version.

For each trial report we first examined whether specific items related to the methodological aspects of randomised trials were reported; based on items in the CONSORT checklist ${ }^{8}$; on average this step took between 15 and 20 minutes per trial report. We then examined any changes in reporting of these items between the original and final version of the manuscript. We classified changes as being items added (item new to final version, not in original version of manuscript), subtracted (item removed from final version, was in original version of manuscript), or altered (item in both original and final version of manuscript, but wording changed). By accessing the prepublication history from each report we then assessed each of the peer reviewer's comments about reporting of methodological items and how authors responded to these requests for each round of the peer review process. In particular we examined comments relating to the reporting of trial design (randomisation and blinding), sample size, details of primary and secondary outcomes, requests for additional analyses, and the trial conclusion. We classified the nature of the comments as having either a positive impact on reporting (comment by peer reviewer judged to have beneficial effect on reporting) or negative impact (comment by peer reviewer judged to have harmful effect on reporting). We were unable to assess any additional comments made by the academic editors as these reports were typically not published alongside the peer reviewers' comments.

\section{Data analysis}

We summarised the characteristics of the studies and details of peer review. The primary analysis focused on the nature and extent of changes made to manuscripts after peer review, in relation to the reporting of methodological aspects of randomised trials, measured as the number and type of items added, subtracted, or altered in the final submitted version of the manuscript. We also carried out a descriptive analysis of the type of methodological changes requested in the peer reviewers' comments and the extent to which authors responded to these requests in their manuscript revision.

\section{Results}

The PubMed publication type search term "Randomized Controlled Trial" identified 513 possible reports of such trials published in the $B M C$-series medical journals in the specified period. After screening the titles and abstracts of all retrieved citations, we reviewed 140 full text articles, resulting in 103 primary reports of randomised trials (figure $\downarrow$ ); we excluded 10 as no prepublication history was available electronically (for technical reasons) at the time of performing this study. This resulted in 93 primary reports of randomised trials (see supplementary appendix 2 for list of included $B M C$-series journals, and appendix 3 for list of included trials) where all submitted versions of the manuscript and the corresponding peer review comments and author responses were available online from the respective $B M C$-series medical journal websites. 


\section{Characteristics of primary reports of randomised trials}

Table $1 \Downarrow$ provides information on the general characteristics of the primary reports of randomised trials as described in the published article. The majority of reports were single centre $(n=47 ; 51 \%)$, parallel group trials $(n=75 ; 81 \%)$, with two study groups $(\mathrm{n}=75 ; 81 \%)$ and a median sample size of 132 participants in each trial (10th to 90 th centile, 30 to 527). Around a third $(n=35 ; 38 \%)$ of reports assessed counselling or lifestyle interventions, $27 \%(\mathrm{n}=25)$ drug interventions, and $14 \%(\mathrm{n}=13)$ surgical interventions. The remaining studies $(n=20,21 \%)$ assessed other types of interventions, such as educational strategies or use of equipment.

\section{Peer reviewers and numerical changes to manuscripts}

By accessing the prepublication history for each published article we were able to record information on the life cycle of each manuscript (table $2 \Downarrow$ ). The median time interval between the original and final submitted version of a manuscript was 148 days (range 29-240) with 36 days (range 4-142) from final submission to online publication. The number of submitted versions of a manuscript varied (median 3, range 2-8), with a median of two peer reviewers (range 1-5) and two review rounds of peer review for each manuscript (range 1-4). Overall, the median proportion of words deleted from the original manuscript was $11 \%$ (range 1-60\%) and for words added was $20 \%$ (range $2-88 \%)$.

\section{Comparison between the original and final submitted manuscript}

Table $3 \Downarrow$ shows the extent of changes in the reporting of methodological details made to manuscripts after peer review. More than half of the articles did not adequately report the results for the primary or secondary outcomes (for example, the estimated effect size and confidence interval $)(n=51 ; 55 \%)$, whether the study was blinded $(n=46 ; 50 \%)$, concealment of the random allocation sequence $(n=50 ; 54 \%)$, important harms $(n=60 ; 65 \%)$, or details of the trial protocol $(n=84 ; 90 \%)$, in either the original or the final version of the manuscript. More than one third of articles also failed to describe the method of random sequence generation $(n=35 ; 38 \%)$, report a sample size calculation $(n=32 ; 34 \%)$, or clearly specify the primary and secondary outcomes $(\mathrm{n}=33 ; 35 \%)$.

Where there were changes between manuscript versions these usually involved "adding" new information that had been missing from the original submission, or "altering" existing information. Changes included adding information about the random sequence generation $(\mathrm{n}=11 ; 12 \%)$ and concealment of allocation sequence $(n=9 ; 10 \%)$, blinding $(n=11 ; 12 \%)$, sample size $(n=9 ; 10 \%)$, a CONSORT flow diagram $(n=9 ; 10 \%)$, changes to tables $(n=17 ; 18 \%)$ or figures $(n=14 ; 15 \%)$, details of trial registration $(n=22 ; 24 \%)$, or adding additional analyses $(n=19 ; 20 \%)$ recommended or requested by the peer reviewer. Other changes included altering information about the trial primary $(n=13 ; 14 \%)$ or secondary $(n=11 ; 12 \%)$ outcomes in the methods section, altering the presentation of the results for the primary $(n=9 ; 10 \%)$ or secondary $(n=8 ; 9 \%)$ outcomes, or altering the trial conclusions $(n=30 ; 32 \%)$. In addition, several items were omitted from the final manuscript, these included "randomised" in the manuscript title $(\mathrm{n}=3)$, one or more study authors $(n=3)$, sample size calculations $(n=2)$, tables $(n=3)$, and figures $(n=4)$. Few changes were made to the abstract, those that were made concerned altering of the abstract conclusion $(n=15 ; 16 \%)$.

\section{Nature of changes requested by peer reviewers and impact on reporting}

In the final part of this study we looked at the nature of changes requested by peer reviewers and the extent to which authors responded to these requests (table $4 \Downarrow$ ). In around a third of manuscripts peer reviewers commented on the trial design (for example, the method of randomisation, blinding) $(n=29 ; 31 \%)$, sample size $(n=30 ; 32 \%)$, and trial conclusions $(n=30 ; 32 \%)$; $24 \%(n=22)$ commented on the primary or secondary outcomes and $16 \%(n=15)$ on the outcome results. In $22 \%(n=20)$ of the manuscripts, peer reviewers recommended or requested additional analyses, $22 \%(\mathrm{n}=20)$ mentioned the CONSORT statement, $5 \%(\mathrm{n}=5)$ mentioned the trial protocol, and $12 \%$ $(n=11)$ mentioned trial registration.

The number of changes requested by peer reviewers was relatively small and most were classified as having a positive impact on the reporting of the final manuscript (table $5 \Downarrow$ ). For example, where peer reviewers commented on the method of randomisation or blinding, the authors either added the item $(n=19)$ or gave more details on how it was done $(n=8)$. Other positive changes included the adding $(n=7)$ or justification $(n=8)$ of the sample size, clarification of the primary and secondary outcomes $(n=5)$ and how they were measured $(n=11)$, clarification $(n=12)$ or the addition $(n=2)$ of the outcomes results, and toning down the conclusion to reflect the trial results $(n=27)$. In some instances peer reviewers' comments had no impact on reporting, in that the authors ignored the comments or responded in the "response to reviewers" without making a change in the manuscript $(n=16)$. For example, the peer reviewer commented on the lack of or small sample size $(n=11)$, clarification of how the primary outcome was measured $(n=3)$, or details on randomisation $(n=2)$, to which the authors either did not respond to the peer reviewers request or responded but said that they did not do it, which was not reflected in the final manuscript.

However, some of the changes requested by peer reviewers were classified as having a negative impact on reporting. This was particularly apparent in the analysis where authors added new subgroup or sensitivity analyses $(n=15)$ requested by the peer reviewer (that is, were not part of the original study and were not reported as such in the final version of the manuscript). Other negative changes included deleting $(n=2)$ or adding retrospective $(n=2)$ sample size calculations, adding a new secondary outcome $(n=2)$, and over-inflation of the conclusion that did not reflect the trial results $(n=3)$. In addition there were two instances where the peer reviewers had requested that the authors change the primary outcome $(n=1)$ or add additional analyses $(n=1)$, and the authors responded to say that was not the purpose of their study. Supplementary appendix 4 provides examples of the type of changes requested by peer reviewers and the authors' response to these requests.

\section{Discussion}

This sample of $B M C$-series medical journals offered a unique opportunity to investigate the effects of peer review on the reporting of published manuscripts, something which for most journals remains hidden within the editorial decision making process. ${ }^{9}$ In our study, the number of changes between the original and final submitted version of a manuscript was relatively small. Most involved adding new information (where this had been missing from the original submission) or altering existing information to give more detail about a specific item. 
The majority of changes recommended or requested by peer reviewers were classified as having a positive impact on reporting, such as adding information about randomisation, blinding, and sample size, altering the trial outcomes (for example, by specifying how the outcome was measured or at what time point), the presentation of the trial results, and the toning down of the conclusion to reflect the trial results. ${ }^{10}$ Some of the changes, however, were classified as having a negative impact on reporting, such as requests by peer reviewers for unplanned additional analyses that were not prespecified in the original trial protocol and that were not reported as such in the final manuscript. There is a concern that these additional analyses could be driven by an existing knowledge of the data, or the interests of the reader, rather than the primary focus of the study. ${ }^{11}$ Importantly, despite some improvements in reporting after peer review, it is clear that peer reviewers often fail to detect several important deficiencies in the reporting of the methods and results of randomised trials. The extent to which these findings are generalisable to other journals with different editorial and peer review processes is unclear. However, the problem of poor reporting is something that is seen consistently across the medical literature, ${ }^{12}$ suggesting that the findings seen in this sample of journals might be representative of other journals.

\section{Comparison with other studies}

We are not aware of other studies specifically dealing with the type and nature of changes made to manuscripts after peer review in the reporting of methodological aspects of randomised trials. Other studies on the impact of peer review have predominantly looked at the use of checklists to improve the quality of peer review, the effects of blinding authors and peer reviewers, ${ }^{13}{ }^{14}$ and the implementation of training strategies for peer reviewers, ${ }^{15}{ }^{16}$ with little empirical evidence to support the use of editorial peer review as a mechanism to ensure quality of biomedical research. ${ }^{5}$ More recently, two randomised controlled trials have shown that adding a statistical reviewer as part of the peer review process has a positive effect on the quality of the final manuscript, ${ }^{17}{ }^{18}$ and that additional review based on reporting guidelines such as STROBE (strengthening the reporting of observational studies in epidemiology) and CONSORT can also improve the quality of submitted manuscripts. ${ }^{19}$

\section{Limitations of this study}

Our study has several limitations. Firstly, our sample was limited to $B M C$-series medical journals where peer reviews are published and available in the public domain. It is unclear the extent to which open peer review hinders reviewers from being overly critical or demanding and whether journals with a closed system might see more changes. Secondly, it is possible that manuscripts published in journals with different editorial and peer review processes, such as journals with a higher impact, greater editorial control, and more resources, may show a greater number of changes after peer review. ${ }^{9}$ Thirdly, we do not know the level of methodological expertise of the peer reviewers and whether they were predominately from a clinical, trial methodology, or statistical background. Finally, we only assessed the effects of peer review on the reporting of methodological aspects of randomised trials. We did not look at the clinical aspects of peer review as this would have required content expertise in each of the specific disease areas under investigation. We are therefore unable to comment on the effect of peer review on improving the reporting of clinical aspects of randomised trials.

\section{Implications for peer review}

This sample of $B M C$-series medical journals, whereby all peer reviewers' comments, authors' responses, and submitted versions of the manuscript are included alongside the published article provides a unique insight into the editorial process. This information is essential to enable readers to have a clear and transparent account of the peer review process. We would strongly recommend this model should be followed by other leading journals.

In our study it is clear that not all changes requested by peer reviewers are appropriate, as authors sometimes acceded to requests for inappropriate revisions to their manuscript; presumably in the hope that it would increase the likelihood of the manuscript being accepted for publication if they made the changes. Getting good peer reviewers for manuscripts is a common problem, particularly for smaller "lower impact" journals. Peer reviewers who do not engage with the manuscript and do it merely to say they review for a particular journal is equally as harmful as an author who fails to provide full details in how the study was carried out. Journal editors need to have better mechanisms to ensure that comments by peer reviewers are appropriate ${ }^{20}$ and that authors respond appropriately. Editors should also consider peer reviewers who are dedicated to reviewing different elements of the manuscript. ${ }^{18}$ The use of checklists and reporting guidelines (such as the CONSORT statement which aims to improve the completeness and transparent reporting of randomised trials) has been shown to help peer reviewers to identify problems in the reporting of manuscripts more effectively. ${ }^{19}$ In our study it took on average between 15 and 20 minutes to review each manuscript against the CONSORT checklist items; a task that could be done internally by a trained editorial assistant (internal peer review) before sending a manuscript out for external peer review. It is extremely unlikely that the "average" peer reviewer has the relevant clinical and methodological expertise to perform each task effectively. ${ }^{21}$

\section{Conclusion}

Based on this sample of $B M C$-series medical journals, we found that peer reviewers failed to detect important deficiencies in the reporting of the methods and results in randomised trials. The number of changes requested by peer reviewers was relatively small; however, most authors did comply with recommendations or requests by peer reviewers in their revised manuscript. The majority of requests could be seen to have a positive impact on reporting, although there were instances of suggestions that were inappropriate and had a negative impact on the reporting of the final manuscript. Better use and adherence of reporting checklists (www.equator-network.org) by journal editors, peer reviewers, and authors could be one important step towards improving the reporting of published articles.

Contributors: SH and DA were involved in the design, implementation, and analysis of the study, and in writing the final manuscript. GC, LY, $\mathrm{JC}, \mathrm{MS}, \mathrm{RW}, \mathrm{IB}$, and LS were involved in the implementation of the study and in commenting on drafts of the final manuscript. $\mathrm{SH}$ is responsible for the overall content as guarantor.

Funding: This study received no external funding.

Competing interests: All authors have completed the ICMJE uniform disclosure form at www.icmje.org/coi_disclosure.pdf and declare: no support from any organisation for the submitted work; no financial relationships with any organisations that might have an interest in the submitted work in the previous three years; no other relationships or activities that could appear to have influenced the submitted work. 


\section{What is already known on this topic}

Despite the widespread use of peer review little is known about its impact on the quality of reporting of published research articles Inadequacies in the methodology and reporting of research is widely recognised

Substantial uncertainty exists about the peer review process as a mechanism to improve reporting of the scientific literature

\section{What this study adds}

Peer reviewers often fail to detect important deficiencies in the reporting of the methods and results of randomised trials Peer reviewers requested relatively few changes for reporting of trial methods and results

Most requests had a positive impact on reporting but in some instances the requested changes could have a negative impact

Data sharing: No additional data available.

Transparency: The lead author (the manuscript's guarantor) affirms that the manuscript is an honest, accurate, and transparent account of the study being reported; that no important aspects of the study have been omitted; and that any discrepancies from the study as planned (and, if relevant, registered) have been explained.

1 International Committee of Medical Journal Editors. Uniform requirement for manuscripts submitted to biomedical journals. 2008. www.icmje.org.

2 Hames I. Peer review and manuscript management in scientific journals: guidelines for good practice. Blackwell, 2007.

3 Rennie D. Suspended judgment. Editorial peer review: let us put it on trial. Control Clin Trials 1992;13:443-5.

4 Smith R. Opening up BMJ peer review. BMJ 1999;318:4-5.

5 Jefferson T, Rudin M, Brodney FS, Davidoff F. Editorial peer review for improving the quality of reports of biomedical studies. Cochrane Database Syst Rev 2007;2:MR000016.

6 Turner L, Shamseer L, Altman DG, Schulz KF, Moher D. Does use of the CONSORT statement impact the completeness of reporting of randomised controlled trials published in medical journals? A Cochrane review. Syst Rev 2012;1:60.

7 BMC Series Journals: peer review process. 2014. www.biomedcentral.com/authors/ bmcseries.

8 Moher D, Hopewell S, Schulz KF, Montori V, Gotzsche PC, Devereaux PJ, et al. CONSORT 2010 explanation and elaboration: updated guidelines for reporting parallel group randomised trials. BMJ 2010;340:c869.

9 Dickersin K, Ssemanda E, Mansell C, Rennie D. What do the JAMA editors say when they discuss manuscripts that they are considering for publication? Developing a schem for classifying the content of editorial discussion. BMC Med Res Methodol 2007;7:44.

10 Boutron I, Dutton S, Ravaud P, Altman DG. Reporting and interpretation of randomized controlled trials with statistically nonsignificant results for primary outcomes. JAMA 2010;303:2058-64.

11 Sun X, Briel M, Busse JW, You JJ, AkI EA, Mejza F, et al. Credibility of claims of subgroup effects in randomised controlled trials: systematic review. BMJ 2012;344:e1553.

12 Hopewell S, Dutton S, Yu LM, Chan AW, Altman DG. The quality of reports of randomised trials in 2000 and 2006: comparative study of articles indexed in PubMed. BMJ 2010;340:c723.
13 Alam M, Kim NA, Havey J, Rademaker A, Ratner D, Tregre B, et al. Blinded vs. unblinded peer review of manuscripts submitted to a dermatology journal: a randomized multi-rater study. Br J Dermatol 2011;165:563-7.

14 Cho MK, Justice AC, Winker MA, Berlin JA, Waeckerle JF, Callaham ML, et al. Masking author identity in peer review: what factors influence masking success? PEER Investigators. JAMA 1998;280:243-5.

15 Schroter S, Black N, Evans S, Godlee F, Osorio L, Smith R. What errors do peer reviewers detect, and does training improve their ability to detect them? $J R$ Soc Med 2008;101:507-14.

16 Schroter S, Black N, Evans S, Carpenter J, Godlee F, Smith R. Effects of training on quality of peer review: randomised controlled trial. BMJ 2004;328:673.

17 Arnau C, Cobo E, Ribera JM, Cardellach F, Selva A, Urrutia A. [Effect of statistical review on manuscript quality in Medicina Clinica (Barcelona): a randomized study]. Med Clin (Barc) 2003;121:690-4.

18 Cobo E, Selva-O'Callagham A, Ribera JM, Cardellach F, Dominguez R, Vilardell M. Statistical reviewers improve reporting in biomedical articles: a randomized trial. PLOS One 2007;2:e332.

19 Cobo E, Cortes J, Ribera JM, Cardellach F, Selva-O'Callaghan A, Kostov B, et al. Effect of using reporting guidelines during peer review on quality of final manuscripts submitted to a biomedical journal: masked randomised trial. BMJ 2011;343:d6783.

20 Kravitz RL, Franks P, Feldman MD, Gerrity M, Byrne C, Tierney WM. Editorial peer reviewers' recommendations at a general medical journal: are they reliable and do editors care? PLoS One 2010;5:e10072.

21 Black N, van RS, Godlee F, Smith R, Evans S. What makes a good reviewer and a good review for a general medical journal? JAMA 1998;280:231-3.

Accepted: 12 June 2014

\section{Cite this as: BMJ 2014;348:g4145}

This is an Open Access article distributed in accordance with the Creative Commons Attribution Non Commercial (CC BY-NC 3.0) license, which permits others to distribute, remix, adapt, build upon this work non-commercially, and license their derivative works on different terms, provided the original work is properly cited and the use is non-commercial. See: http://creativecommons.org/licenses/by-nc/3.0/. 


\section{Tables}

Table 1 | General characteristics of randomised trials published in BMC-series medical journals in 2012

\begin{tabular}{ll} 
Characteristics & No (\%) of manuscripts ( $\mathbf{n} \mathbf{9 3})$ \\
Trial design: & $75(81)$ \\
\hline Parallel & $7(7.5)$ \\
\hline Crossover & $9(9.5)$ \\
\hline Cluster & $2(2)$ \\
\hline Other & $25(27)$ \\
\hline Intervention: & $13(14)$ \\
\hline Drug & $35(38)$ \\
\hline Surgery or procedure & $20(21)$ \\
\hline Counselling or lifestyle & $47(51)$ \\
\hline Other* & $30(32)$ \\
\hline Study centres: & $16(17)$ \\
\hline Single & $75(81)$ \\
\hline Multiple & $9(9.5)$ \\
\hline Unclear & $9(9.5)$ \\
\hline No of study groups & $132(30$ to 527$)$ \\
\hline 2 & \\
\hline 3 & \\
\hline$\geq 4$ & \\
\hline Median No of participants per trial (10th to 90th centile) & \\
\hline \multirow{2}{*}{ For example, education, equipment. } & \\
\hline
\end{tabular}


Table 2| Details of peer review and numerical changes to manuscripts ( $n=93)$. Values are medians (ranges), interquartile ranges unless stated otherwise

\begin{tabular}{|c|c|}
\hline Details of peer review & Results \\
\hline \multicolumn{2}{|l|}{ Factors per manuscript: } \\
\hline Median No (range) of peer reviewers & $2(1-5)$ \\
\hline Median No (range) of peer review rounds & $2(1-4)$ \\
\hline Median No (range) of author responses & $2(1-7)$ \\
\hline Median No (range) of submitted versions & $3(2-8)$ \\
\hline Words added (range) to final submitted version (\%) & 20 (2-88), 12-36 \\
\hline Words deleted (range) from final submitted version (\%) & $11(1-60), 5-21$ \\
\hline Time between original and final submitted version (days) & $148(29-240), 105-204$ \\
\hline Time between final submitted and published version (days) & $36(4-142), 23-54$ \\
\hline
\end{tabular}




\begin{tabular}{|c|c|c|c|c|c|}
\hline \multirow[b]{2}{*}{ Items } & \multicolumn{5}{|c|}{ No (\%) } \\
\hline & Reported (no change) ${ }^{*}$ & Not reported (no change) $\dagger$ & Added $\neq$ & Subtracted§ & Altered $\eta$ \\
\hline "Randomised" in title & $71(76)$ & $15(16)$ & $4(4)$ & $3(3)$ & 0 \\
\hline Author names & $87(94)$ & 0 & $3(3)$ & $3(3)$ & 0 \\
\hline \multicolumn{6}{|l|}{ Abstract: } \\
\hline Method of randomisation & $8(9)$ & $84(90)$ & $1(1)$ & 0 & 0 \\
\hline Primary outcome & $22(24)$ & $67(72)$ & $1(1)$ & 0 & $3(3)$ \\
\hline No randomised & $28(30)$ & $64(69)$ & $1(1)$ & 0 & 0 \\
\hline No analysed & $14(15)$ & $79(85)$ & 0 & 0 & 0 \\
\hline Conclusion & $78(84)$ & 0 & 0 & 0 & $15(16)$ \\
\hline \multicolumn{6}{|l|}{ Methods: } \\
\hline Sequence generation & $44(47)$ & $35(38)$ & $11(12)$ & 0 & $3(3)$ \\
\hline Allocation sequence & $32(34)$ & $50(54)$ & $9(10)$ & 0 & $2(2)$ \\
\hline Blinding & $31(33)$ & $46(50)$ & $11(12)$ & 0 & $5(5)$ \\
\hline Primary outcome & $47(51)$ & $33(35)$ & 0 & 0 & $13(14)$ \\
\hline Secondary outcomes & $47(51)$ & $33(35)$ & $2(2)$ & 0 & $11(12)$ \\
\hline Sample size & $42(45)$ & $32(34)$ & $9(10)$ & $2(2)$ & $8(9)$ \\
\hline \multicolumn{6}{|l|}{ Results: } \\
\hline Flow diagram & $52(56.5)$ & $25(27)$ & $9(10)$ & 0 & $6(6.5)$ \\
\hline No randomised & $77(83)$ & $8(9)$ & $6(6)$ & 0 & $2(2)$ \\
\hline No analysed & $73(78)$ & $14(15)$ & $5(5)$ & 0 & $1(1)$ \\
\hline Primary outcome & $33(35)$ & $51(55)$ & 0 & 0 & $9(10)$ \\
\hline Secondary outcomes & $31(33)$ & $51(55)$ & $3(3)$ & 0 & $8(9)$ \\
\hline Additional analyses & $9(10)$ & $65(70)$ & $19(20)$ & 0 & \\
\hline Tables (important changes) & $56(60)$ & $3(3)$ & $17(18)$ & $3(3)$ & $14(15)$ \\
\hline Figures (important changes) & $56(60)$ & $15(16)$ & $14(15)$ & $4(4)$ & $4(4)$ \\
\hline Harms & $28(30)$ & $60(65)$ & $2(2)$ & 0 & $3(3)$ \\
\hline Conclusions & $63(68)$ & 0 & 0 & 0 & $30(32)$ \\
\hline \multicolumn{6}{|l|}{ Other information: } \\
\hline Funding & $67(72)$ & $15(16)$ & $5(5)$ & 0 & $4(4)$ \\
\hline Trial protocol & $8(9)$ & $84(90)$ & $1(1)$ & 0 & 0 \\
\hline Trial registration & $47(51)$ & $21(23)$ & $22(24)$ & $1(1)$ & $2(2)$ \\
\hline
\end{tabular}

*Item reported in both original and final submitted version of manuscript (no change on wording). †ttem not reported in either original or final submitted version of manuscript.

\#ltem new to final submitted version, was not in original version of manuscript.

§ltem removed from final submitted version, was in original version of manuscript.

flltem in both original and final submitted version of manuscript but wording changed (usually involved giving more detail for a specific item). 
Table 4 | Nature of changes requested by peer reviewers (per manuscript) and impact on reporting

No (\%)

Nature of changes Manuscripts $(n=93)$ Positive impact* No impact on reportingt No impact on reporting§ Negative impact

\begin{tabular}{|c|c|c|c|c|c|}
\hline Abstract conclusion & $15(16)$ & $14(93)$ & 0 & 0 & $1(7)$ \\
\hline Trial design (randomisation and blinding) & $29(31)$ & $27(93)$ & $2(7)$ & 0 & 0 \\
\hline Sample size & $30(32)$ & $15(50)$ & $11(37)$ & 0 & $4(13)$ \\
\hline Primary and secondary outcomes: methods & $22(24)$ & $16(73)$ & $3(13)$ & $1(5)$ & $2(9)$ \\
\hline Primary and secondary outcomes: results & $15(16)$ & $14(93)$ & 0 & 0 & $1(7)$ \\
\hline Additional analyses & $20(22)$ & $4(20)$ & 0 & $1(5)$ & $15(75)$ \\
\hline Conclusion & $30(32)$ & $27(90)$ & 0 & 0 & $3(10)$ \\
\hline
\end{tabular}

*Peer reviewers' comments judged to have beneficial effect on reporting, and author made change.

†Peer reviewers' comments judged to have beneficial effect on reporting, and author did not make change.

§Peer reviewers' comments judged to have harmful effect on reporting, and author did not make change.

IPeer reviewers' comments judged to have harmful effect on reporting, and author made change. 


\begin{tabular}{|c|c|c|c|c|}
\hline Item & Positive impact ${ }^{*}$ & No impact† & No impact‡ & Negative impact§ \\
\hline Abstract conclusion & $\begin{array}{l}\text { PR: conclusions do not reflect results. AU: } \\
\text { toned down conclusion }(n=14)\end{array}$ & & & $\begin{array}{l}\text { PR: revise conclusions. AU: inflated } \\
\text { conclusion not reflecting results }(n=1)\end{array}$ \\
\hline Trial design & $\begin{array}{l}\text { PR: no details on randomisation/blinding. } \\
\text { AU: added item }(n=19) \text {. PR: clarify details } \\
\text { on randomisation/blinding. AU: gave more } \\
\text { detail }(n=8)\end{array}$ & $\begin{array}{l}\text { PR: explain randomisation. AU: } \\
\text { added text but did not } \\
\text { understand concept }(n=2)\end{array}$ & & \\
\hline Sample size & $\begin{array}{l}\text { PR: no sample size. AU: added item }(n=7) \text {. } \\
\text { PR: justify sample size. AU: gave more } \\
\text { detail }(n=8)\end{array}$ & $\begin{array}{l}\text { PR: no sample size. AU: did not } \\
\text { response or said did not do one } \\
(n=6) \text {. PR: small sample size. } \\
\text { AU: justified sample size in } \\
\text { response but not in manuscript } \\
(n=4) \text {. PR: small sample size. } \\
\text { AU: added to limitations section } \\
(n=1)\end{array}$ & & $\begin{array}{l}\text { PR: justify how sample done. AU: } \\
\text { deleted sample size }(n=2) \text {. PR: justify } \\
\text { sample size. AU: added post hoc } \\
\text { sample size, but was not reported as } \\
\text { such in manuscript }(n=2)\end{array}$ \\
\hline $\begin{array}{l}\text { Primary and secondary } \\
\text { outcomes measured }\end{array}$ & $\begin{array}{l}\text { PR: clarify which primary and secondary } \\
\text { outcomes. AU: gave more detail }(n=5) \text {. } \\
\text { PR: how measured. AU: gave more detail } \\
(n=11)\end{array}$ & $\begin{array}{l}\text { PR: clarify how primary outcome } \\
\text { measured. AU: did not respond } \\
(n=3)\end{array}$ & $\begin{array}{l}\text { PR: change primary } \\
\text { outcome. AU: said no } \\
\text { as not primary outcome } \\
\text { for the study }(n=1)\end{array}$ & $\begin{array}{l}\text { PR: add new secondary outcome. AU: } \\
\text { added new outcome in methods }(n=2)\end{array}$ \\
\hline $\begin{array}{l}\text { Primary and secondary } \\
\text { outcomes results }\end{array}$ & $\begin{array}{l}\text { PR: clarify results. AU: gave more detail } \\
(n=12) \text {. PR: no results. AU: added results } \\
\text { for secondary outcome }(n=2)\end{array}$ & & & $\begin{array}{l}\text { PR: add new secondary outcome. AU: } \\
\text { added new outcome in results }(n=1)\end{array}$ \\
\hline Additional analyses & $\begin{array}{l}\text { PR: clarify analysis. AU: added results for } \\
\text { comparison across groups }(n=4)\end{array}$ & & $\begin{array}{l}\text { PR: add new additional } \\
\text { analyses. AU: said no } \\
\text { was not purpose of the } \\
\text { study ( } n=1 \text { ) }\end{array}$ & $\begin{array}{l}\text { PR: add new additional analyses. AU: } \\
\text { added subgroup/sensitivity analysis } \\
(n=15)\end{array}$ \\
\hline Conclusion & $\begin{array}{l}\text { PR: conclusions do not reflect results. AU: } \\
\text { toned down conclusion }(n=27)\end{array}$ & & & $\begin{array}{l}\text { PR: revise conclusions. AU: inflated } \\
\text { conclusion but did not reflect results } \\
(n=3)\end{array}$ \\
\hline
\end{tabular}

*Peer reviewers' comments judged to have beneficial effect on reporting, and author made change.

†Peer reviewers' comments judged to have beneficial effect on reporting, and author did not make change.

$\ddagger$ Peer reviewers' comments judged to have harmful effect on reporting, and author did not make change.

$\S$ Peer reviewers' comments judged to have harmful effect on reporting, and author made change. 


\section{Figure}

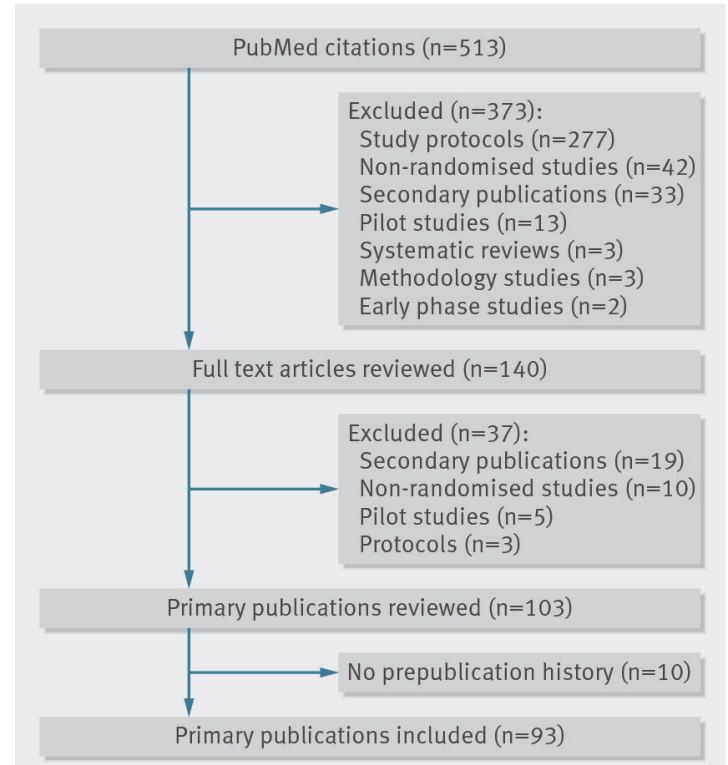

Identification of randomised trials published in $B M C$-series medical journals from PubMed citations indexed from January to December 2012 